\title{
A Queueing Theoretic Approach for Performance Prediction of Collaborative Active Protection Systems
}

\author{
Graham V. Weinberg ${ }^{a}$ \\ ${ }^{a}$ Defence Science and Technology Group, Joint and Operations Analysis Division, Edinburgh, South \\ Australia, 5111, Australia \\ Email: Graham.Weinberg@dst.defence.gov.au
}

\begin{abstract}
The defence of a team of armoured fighting vehicles (AFVs) against both military and improvised threats is of considerable importance to defence agencies. Active protection systems (APS) equip a vehicle with both automatic threat detection as well as countermeasures. A concept of significant contemporary interest is that of collaboration between vehicles equipped with APS. In this context, collaboration permits information on threat detection and tracking to be shared amongst members of the combat team. This then allows threat countermeasures to be both scheduled and then activated.
\end{abstract}

A series of studies conducted by Defence Science and Technology Group (DSTG) have generated a number of stochastic models to examine this concept. In the first instance a discretised time model was produced, which then allowed performance prediction of collaborative APS (C-APS) to be undertaken. A limitation of this approach is that threats may arrive at any point between discrete time epochs, and hence the discretised model does not reflect observable reality. Hence a second analysis investigated the application of jump stochastic processes to undertake performance prediction in continuous time. This approach provided quantitative performance prediction when the AFV is subjected to up to two threats. When the number of threats exceeds two the analytic solution increases in complexity and becomes difficult to utilise, justifying the search for an alternative performance prediction methodology.

In order to produce a manageable framework to conduct C-APS performance prediction in the presence of more than two threats, a queueing theoretic approach has been formulated. This scheme views the vehicles with effectors as a network of queues, where a threat is interpreted as a customer arriving into a particular queue. The process of elimination of the threat is the customer's service time in the respective queue. This formulation of the C-APS performance prediction problem then yields a useful expression for the probability that threats are neutralised by a given time. Consequently this provides a mechanism to assess the performance of various configurations of C-APS.

Due to the fact that directed energy weapons (DEWs) are of contemporary interest for threat defeat with AFVs, the specific model performance prediction examples will utilise C-APS when the countermeasure is provided by high energy laser (HEL) DEWs.

Keywords: Queueing models, armoured fighting vehicles, active protection systems, collaborative defence, performance prediction 


\section{INTRODUCTION}

Armoured fighting vehicles (AFVs) operate in a hostile environment with threats resulting from both direct engagement with enemy forces, as well as attacks by insurgents with improvised threats. As a consequence of this, AFVs require self-defence measures to be integrated into their design. There are physical limits to the practicality of armour on combat vehicles, and therefore it is critical that defence be provided by a more active mechanism. Consequently, active protection systems (APS) have been designed to provide an AFV with defence against airborne threats, such as rocket propelled grenades (RPGs) and drones equipped with an explosive charge. For discussions on the design and utility of APS one can consult Meyer (1998) and Feickert (2016). An APS applies a sensing tool to detect the presence of threats, and then utilises a countermeasure to disrupt or destroy the threat, as explained in Yang and Xu (2020). Sensing can be provided by radar or laser based detection measures. Countermeasures for APS are classified into two groups. The first is referred to as soft kill measures, which are designed to alter the AFVs signature in a given spectrum (electromagnetic and acoustic, for example). As such, these countermeasures protect the vehicle by deceiving the threat as to its target's true location. The second class of countermeasures are referred to as hard kill, and are designed to disrupt or destroy the threat directly, through the result of direct fire engagement. Although APS has provided AFVs with an automated defence mechanism it is important to note that they do not provide invincible countermeasures, as discussed in Graswald et al. (2019). Depending on the sensor's field of view it is possible that a threat can be launched from an angle relative the the AFV that obscures its trajectory. Hence in this study it will be assumed that the engagement between the AFV team and the threats it faces is within the same field of operation and is close combat.

A concept being currently explored at Defence Science and Technology Group (DSTG) is that of collaborative APS (C-APS), where a team of AFVs utilise information sharing and coordination of effectors to enhance their survivability when under attack. This concept has the potential to not only address some of the vulnerabilities of single vehicle APS but is capable of enhancing the survivability of the AFV team in general. The main idea is that the AFV team will consist of a series of vehicles with sensing capability, and also a set of vehicles with countermeasures. This framework also permits each vehicle to be equipped with both capabilities, but also allows there to be vehicles with enhanced capability, such as a more powerful and extensive suite of sensors. Coordination between vehicles is provided by a secure combat cloud, as discussed in Heiss et al. (2019).

The modus operandi of the C-APS scheme is as follows. Vehicles equipped with automatic sensing capability scan the battlespace for evidence of threats. When a threat is detected vehicles with disruption capability can be scheduled to counter the threat. Scheduling may be based upon determination of the closest asset with disruption capability and asset availability.

Figure 1 provides an illustration of the combat scene under consideration. The AFV team consists of five members, denoted $B_{1}, B_{2}, \ldots, B_{5}$, while there are three threats to the combat team, denoted $V_{1}(t), V_{2}(t)$ and $V_{3}(t)$ respectively. An assumption adopted for analytic tractibility is that the AFV team members remain stationary during the engagement. The fact that the threats move with time is indicated through their dependence on $t$. In this scenario, $B_{1}$ is targeted by $V_{1}(t), B_{3}$ is targeted by $V_{2}(t)$ and $B_{4}$ is being targeted by $V_{3}(t)$. The three vehicles targeted by threats are assumed to be operating sensing capabilities, and consequently may have been detected by an enemy's radio frequency (RF) seeker. From a tactical perspective, $B_{2}$ has the best line of sight defence for each of the targeted vehicles, while the other AFV team members will only have line of sight strike capability for subintervals of time that it takes for a threat to reach its target. Otherwise there is a risk of collateral damage to the AFV team.

The primary objective of the modelling efforts at DSTG has been to produce a stochastically driven representation of the scenario in Figure 1 which permits the probability of threat defeat to be quantified in terms of permutations of AFVs with target detection and disruption capability. The underlying model framework is common across a number of modelling scenarios. For example, the defence of a fleet of maritime platforms from missile threats has resulted in similar analysis, as illustrated in Perry et al. (2002). There have also been a number of studies of APS performance prediction, with Feng et al. (2020) being an example where the probability of threat defeat is expressed in terms of a number of combat team attributes and defensive capabilities.

DSTG's initial work in quantifying AFV survivability with C-APS has been documented in Weinberg (2021), where a discretised time approach was adopted. This study also specialised the countermeasure to a high power radio frequency (HPRF) DEW; useful outlines of the principles of DEW include Nielsen (1994) and Deveci (2007). HPRF DEW countermeasures have utility in disabling a missile or drone's guidance, navigation and 


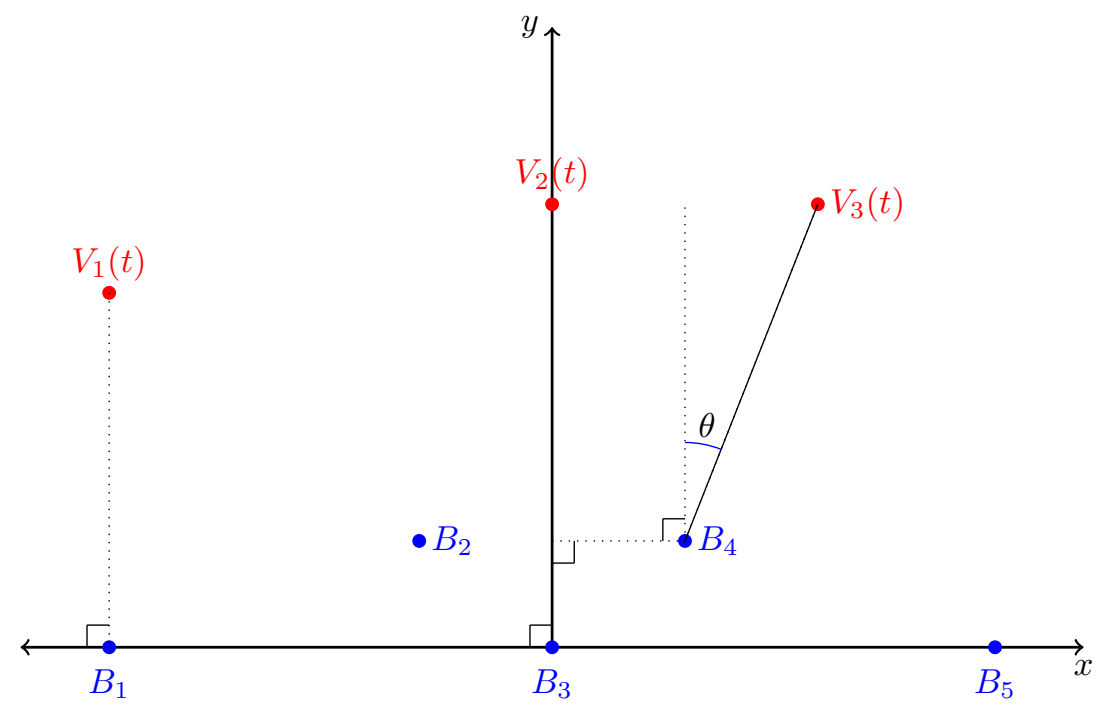

Figure 1. An illustration of the combat scenario under consideration. An AFV team consisting of five members $\left(B_{1}, B_{2}, \ldots, B_{5}\right)$ is facing a series of threats $\left(V_{1}(t), V_{2}(t), V_{3}(t)\right)$. Throughout the engagement the AFV team members remain stationary, while the threats are moving, as indicated by their time dependence.

control, as can be inferred from the discussion in Clarke et al. (2011). Hence, in a sense, a HPRF DEW can be interpreted as producing a soft kill option. The issue with the approach in Weinberg (2021) is that it does not account for the fact that a threat may arrive between two discrete time points. Hence it is not an accurate reflection of the observed reality in the combat space. Consequently a second study introduced a continuous time stochastic process to model the number of threats defeated by a given time, as demonstrated in Weinberg and Kracman (2021). The key to this was to model the number of threats defeated by a given time as a jump stochastic process, and to determine the distribution of the sojourn time in states. This produced a simple solution for the case where the AFV team is facing a single threat, while for the situation of two threats the distribution of sojourn times required Monte Carlo estimation to produce results. Countermeasures in Weinberg and Kracman (2021) are provided by high energy laser (HEL) DEWs. The utility of HEL DEWs for AFV defence is documented in Hafften and Stratton (2004) and Lavan (2009). Extension of the approach introduced in Weinberg and Kracman (2021) to the case of more than two threats has proven to be possible but extremely complex. Hence it was necessary to examine whether an alterative approach could be found to quantify AFV team survivability in the presence of multiple threats.

Towards this objective, Section 2 introduces a novel approach to the problem under consideration by viewing C-APS performance prediction from the perspective of a network of queues. For countermeasure purposes the AFV team members with threat disruption capability are viewed as servers in this queueing network. When a threat is detected it is then passed to the next available queue, and the time it spends in the queue is the time it takes for the effector to disable it. If all queues are occupied then detected threats are placed in a holding queue until the next queue is free. It will be shown that this interpretation can yield an analytic solution for the quantification of AFV team performance under the condition that the number of effectors in the AFV team exceeds the number of threats. Relaxing this condition will also be discussed, and as it will become evident, this is possible with this network of queues approach.

\section{Performance Prediction Model}

Suppose that the AFV team consists of $K$ vehicles denoted $\mathcal{T}=\left\{B_{i}, i \in\{1,2, \ldots, K\}\right\}$, and that it is facing a series of $M$ threats, denoted $\mathcal{V}=\left\{V_{j}(t), j \in\{1,2, \ldots, M\}\right\}$. Define the set $\mathcal{D} \subseteq \mathcal{T}$ such that members of $\mathcal{D}$ have a target detection and tracking capability. Additionally let $\mathcal{S} \subseteq \mathcal{T}$ consist of vehicles with a threat disruption capability. These two sets are not necessarily disjoint.

Let $X(t)$ be the number of threats detected by the AFV team by time $t$. Suppose that $\theta_{i}^{(j)}(t)$ is the probability that threat $V_{j}$ is detected by the $i$ th detector located on $B_{i} \in \mathcal{D}$ by time $t$. Then the probability that threat $V_{j}$ is detected by the AFV team by time $t$ is the probability that at least one member of $\mathcal{D}$ detects it. This can be 
shown to be

$$
\mathbf{P}\left(V_{j} \text { detected at time } t\right)=1-\prod_{\left\{i: B_{i} \in \mathcal{D}\right\}}\left(1-\theta_{i}^{(j)}(t)\right),
$$

under the assumption of independent detections. Due to the latter it follows that $X(t)$ will have a PoissonBinomial distribution. However, for the purposes of this analysis, only the probability that all threats are detected by a given time will be required, which under the assumption of independent sensors is a product of (1) for each threat. Hence

$$
\mathbf{P}(X(t)=M)=\prod_{j=1}^{M}\left[1-\prod_{\left\{i: B_{i} \in \mathcal{D}\right\}}\left(1-\theta_{i}^{(j)}(t)\right)\right]
$$

Define $N=\operatorname{card}(\mathcal{S})$ to be the total number of effectors available ${ }^{1}$. One can view the task of threat defeat as a two stage process. When threats are detected, they are then passed onto available effectors to be neutralised. Hence the process of threat defeat can be analysed from a network of queues perspective. As remarked in the Introduction, the effectors are the queue servers and the service time of a threat within a given queue is equivalent to the time it takes to defeat it. This queueing network approach is formulated mathematically as follows.

Let $W_{i}(t)$ be the number of threats in the queue corresponding to effector $i$, where $i \in\{1,2, \ldots, N\}$. Then the random variable

$$
W(t):=\sum_{i=1}^{N} W_{i}(t)
$$

counts the total number of threats being disrupted at time $t$. Hence one can define a random variable

$$
Z(t)=X(t)-\sum_{i=1}^{N} W_{i}(t)
$$

which counts the number of threats neutralised by time $t$, so that $Z(t) \in\{0,1,2, \ldots, M\}$. Note that by conditioning on the number of threats detected by time $t$,

$$
\mathbf{P}(Z(t)=z)=\sum_{k=0}^{M} \mathbf{P}(X(t)=k) \mathbf{P}\left(\sum_{i=1}^{N} W_{i}(t)=k-z \mid X(t)=k\right),
$$

for $0 \leq z \leq M$. Since the sum defined in (3) can never be negative it follows that (5) can be reduced to

$$
\mathbf{P}(Z(t)=z)=\sum_{k=z}^{M} \mathbf{P}(X(t)=k) \mathbf{P}\left(\sum_{i=1}^{N} W_{i}(t)=k-z \mid X(t)=k\right) .
$$

In view of the fact that the interest is in the defeat of all threats by time $t$ one can focus on

$$
\mathbf{P}(Z(t)=M)=\mathbf{P}(X(t)=M) \mathbf{P}\left(\sum_{i=1}^{N} W_{i}(t)=0 \mid X(t)=M\right) .
$$

One can now apply (2) to (7). Evaluation of the second expression in (7) requires careful analysis.

The simplest case to consider is the situation where the number of threats is smaller than or equal to the total number of effectors $(M \leq N)$. In such situations it is always possible to assign a threat to an empty queue. Suppose that $\phi_{i}^{(j)}(t)$ is the probability that threat $V_{j}$ is disrupted by effector $i$ located on AFV $B_{i} \in \mathcal{S}$ by time $t$. Then the probability that this threat is disrupted by the AFV team by time $t$, given it has been detected, is given by

$$
\mathbf{P}\left(V_{j} \text { disrupted by time } t \mid V_{j} \text { detected by the team }\right)=1-\prod_{\left\{i: B_{i} \in \mathcal{S}\right\}}\left(1-\phi_{i}^{(j)}(t)\right) .
$$

\footnotetext{
${ }^{1}$ Card is the cardinality of the given set.
} 


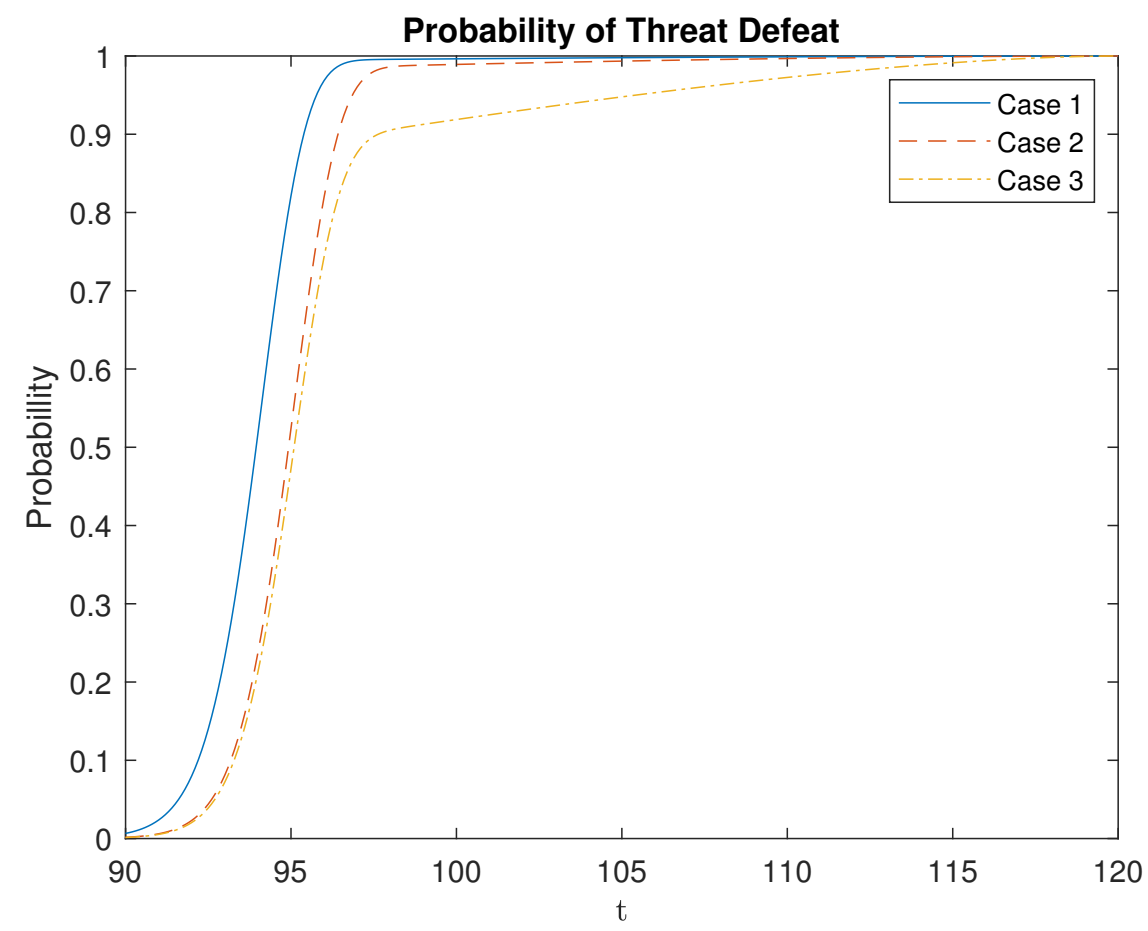

Figure 2. Performance prediction for the three cases. The first case assumes that sensors and effectors are available on each vehicle. Case two supposes that sensing and disruption is provided by the vehicle being targeted. Case 3 is similar to Case 2 except countermeasures are offset, so that defence of a targeted vehicle is provided by an adjacent effector.

Examining the second expression on the right hand side in (7), the probability that all the queues are empty by time $t$, given all the threats have been detected, is the probability that each individual threat is defeated under the condition that it has been detected. Hence, in view of (8),

$$
\mathbf{P}\left(\sum_{i=1}^{N} W_{i}(t)=0 \mid X(t)=M\right)=\prod_{j=1}^{M}\left[1-\prod_{\left\{i: B_{i} \in \mathcal{S}\right\}}\left(1-\phi_{i}^{(j)}(t)\right)\right] .
$$

Finally, by an application of (2) and (9) to (7), one arrives at

$$
\mathbf{P}(Z(t)=M)=\prod_{j=1}^{M}\left\{\left[1-\prod_{\left\{i: B_{i} \in \mathcal{D}\right\}}\left(1-\theta_{i}^{(j)}(t)\right)\right]\left[1-\prod_{\left\{i: B_{i} \in \mathcal{S}\right\}}\left(1-\phi_{i}^{(j)}(t)\right)\right]\right\} .
$$

Hence (10) can be used as a metric to quantify the performance of an AFV team, equipped with C-APS, in the presence of $M$ threats, provided the latter is smaller than the total number of effectors $N$.

It is worth observing that optimal choices for $\mathcal{D}$ and $\mathcal{S}$ can be determined through analysis of (10), where optimality is in the context of maximising the likelihood of total threat defeat. Towards this end, note that if $A \subset B$ are subsets of the natural numbers then $\operatorname{Card}(A)<\operatorname{Card}(B)$ and if $0<\alpha_{k}<1$ then $\prod_{k \in A} \alpha_{k}>$ $\prod_{k \in B} \alpha_{k}$ and therefore $1-\prod_{k \in A} \alpha_{k}<1-\prod_{k \in B} \alpha_{k}$. By applying this to the logarithm of (10), and observing that the logarithm is an increasing function, one can conclude that the probability (10) will be maximised when the sets $\mathcal{D}$ and $\mathcal{S}$ are maximum. Consequently maximal performance under C-APS will be achieved when each AFV is equipped with a sensor and an effector. However, it may not always be practical to activate an effector if there is a risk of collateral damage to the AFV team members.

\section{3 examples of Performance}

The combat scenario depicted in Figure 1 will be the focus of this demonstration of the application of (10). Hence throughout the following it will be assumed that $M=3$ and $K=5$. In terms of Cartesian Geometry, 
suppose that $B_{1}$ is located at $(-30,0), B_{2}$ at $(-9,6), B_{3}$ at the origin, $B_{4}$ at $(9,6)$ and $B_{5}$ at $(30,0)$; in all cases distance is measured in metres. The three threats are assumed to be homogeneous, travelling at the same constant speed of 40 metres per second and taking the common time of 120 seconds to reach their targets. Threats $V_{1}(t)$ and $V_{2}(t)$ travel on a linear path parellel to the $y$-axis; threat $V_{3}(t)$ also travels in a linear path but at an angle of $15^{\circ}$ to the azimuth direction as illustrated in Figure 1. These conditions imply that at time $t, V_{1}(t)$ has coordinates $(-30,40(120-t)), V_{2}(t)$ is at $(0,40(120-t))$ and $V_{3}(t)$ is at $\left(9+40(120-t) \sin \left(15^{\circ}\right), 6+40(120-t) \cos \left(15^{\circ}\right)\right)$.

Three cases will be investigated: the first is for the situation where all five vehicles have a detection capability, while $B_{1}$ and $B_{2}$ are used for defence against $V_{1}(t), B_{3}$ is used to defeat $V_{2}(t)$ and $B_{4}$ and $B_{5}$ are used to counter $V_{3}(t)$. In the second case it will be assumed that only $B_{1}, B_{3}$ and $B_{4}$ are equipped with sensors, and that $B_{1}$ can be used to counter $V_{1}(t), B_{3}$ for $V_{2}(t)$ and $B_{4}$ for $V_{3}(t)$ defeat. The third case also assumes that only $B_{1}, B_{3}$ and $B_{4}$ are equipped with sensors, but examines the situation when $B_{2}$ is used to counter $V_{1}(t)$, $B_{4}$ for defeating $V_{2}(t)$ and $B_{5}$ for disrupting $V_{3}(t)$. Thus case 1 has the maximal coverage in terms of sensors, and also each vehicle is equipped with a disruption capability. Case 2 assumes that the targets of the threat have sensors (and so may have been targeted by an RF seeker), and that each of these vehicles is responsible for its own defence. Case 3 adopts the same detection protocol as for Case 2 but examines the impact of defence of each targeted vehicle when it is provided by an adjacent vehicle.

The probability of detection and defeat will be adopted directly from Weinberg and Kracman (2021). As justified in the latter, the probability of target detection is modelled through the Marcum Q-Function, so that

$$
\theta_{i}^{(j)}(t)=Q_{M}\left(\sqrt{2 M \zeta_{i}^{(j)}(t)}, \sqrt{2 \nu}\right)
$$

where

$$
Q_{M}(a, b)=\frac{1}{a^{M-1}} \int_{b}^{\infty} x^{M} \exp \left(-\frac{\left(x^{2}+a^{2}\right)}{2}\right) I_{M-1}(a x) d x
$$

and $I_{M}$ is the modified Bessel function of the first kind of order $M, \zeta_{i}^{(j)}$ is the signal to clutter ratio (SCR) and $\nu$ is the detection threshold, set to produce a desired false alarm rate. The notation employed here for the SCR indicates that it is for detection of threat $j$ from vehicle $i$. This SCR can be specified as a function of radar characteristics through

$$
\zeta_{i}^{(j)}(t)=\frac{P_{R} G_{R}^{2} \lambda_{R} \mathbf{E}\left(\sigma_{R}\right)}{(4 \pi)^{3}\left(R_{i}^{(j)}(t)\right)^{4} 2 \theta}
$$

where $P_{R}$ is the power radiated by the radar (in Watts), $G_{R}$ is the radar antenna's gain (in dBi), $R_{i}^{(j)}$ is the distance between the $i$ th effector and the target $V_{j}(t), \lambda_{R}$ is the wavelength (in metres) of the radar signal, $\mathbf{E}\left(\sigma_{R}\right)$ is the mean of the radar cross section (RCS) $\sigma_{R}$ of the target (in square metres) and $\theta$ is the variance of the compound Gaussian model in the underlying assumption of Rayleigh amplitude statistics. The latter parameter has been set so that a target with 1 square metre radar cross section can be detected almost certainly within $1 \mathrm{~km}$, when the following radar parameters are adopted: $P_{R}=10^{8}$ Watts with a gain of $G_{R}=100$ $\mathrm{dBi}$ and a wavelength of $\lambda_{R}=0.03$, where the latter corresponds to a frequency of $10 \mathrm{GHz}$. The average RCS has been chosen to be unity, so that $\mathbf{E}\left(\sigma_{R}\right)=1$. It will also be assumed that $M=32$ with a false alarm probability of $10^{-4}$.

A HEL DEW is assumed for each platform with countermeasure capability, as in Weinberg and Kracman (2021). Therefore

$$
\phi_{i}^{(j)}(t)=\frac{\mu_{\tau}}{1-e^{-\mu_{\tau} t}} \int_{0}^{t} e^{-\mu_{\tau} s} e^{-1.8194 \mu_{\sigma} U\left[\int_{t-s}^{t}\left(R_{i}^{(j)}(x)\right)^{-2} d x\right]^{-1}} d s
$$

where $R_{i}^{(j)}(t)$ is defined as in the specification for the radar probability of effect, and the HEL DEW is operating at a wavelength of $\lambda=1.045 \mu \mathrm{m}$ and output power of $30 \mathrm{~kW}$. The reciprocal of $\mu_{\tau}$ is the expected DEW dwell time on the target to cause a disruption and the reciprocal of $\mu_{\sigma}$ is the expected vulnerability area of the target. The HEL DEW disruption threshold of the target is $U$, which is the HEL power level on the target to cause a thermal effect. Further details of the derivation and assumptions adopted for the HEL DEW can be sourced from Weinberg and Kracman (2021). For the purposes of this paper the average dwell time has 
been selected to be 3 seconds and the expected vulnerability area is 1 square metre. The disruption threshold is assumed to be 1 Watt per square metre.

Figure 2 plots the probability of disruption, as provided by (10), for the three cases outlined above. Recall that the three threats travel toward their targets at the same rate. At time $90 \mathrm{~s}$ these threats are $1200 \mathrm{~m}$ away, while at time $100 \mathrm{~s}$ they are $800 \mathrm{~m}$. By time $105 \mathrm{~s}$ the threats are at $600 \mathrm{~m}$. The figure demonstrates that Case 1 has the maximal probability of defeat of the threats, while Case 2 has slightly worse performance. Case 3 has the overall worst performance, and takes considerably more time to defeat the threats with almost certainty. The interesting characteristic of Case 3 is that when time exceeds approximately $97 \mathrm{~s}$ there is a sharp decline in performance relative to the other two cases. The reason for this is likely to be due to the fact that in this offset case the distance between an effector on an AFV and the threat it is countering can never reach zero. Hence there will be a limit to the maximum power on the target, unlike the other two cases.

\section{CONClusions AND Future Work}

The purpose of this study was to produce a performance prediction model for an AFV team, operating with some degree of C-APS, when facing a number of threats. The model assumed that the number of available effectors exceeded the number of threats, and utilised a queueing theory formulation. An example to HEL DEW defence illustrated the utility of the method. The queueing theory approach to this problem is a also suitable way in which to examine the situation when the number of threats exceeds the number of effectors. In order to do this it will be necessary to examine the situation when arriving threats must be delayed before processing in an available queue. This analysis will form the basis for subsequent work at DSTG.

\section{REFERENCES}

Clarke, T., A. Taylor, N. Estep, S. Yakura, D. Brumit, D. Dietz, S. Hemmady, and J. Duffet (2011). Predictive modeling of high-power electromagnetic effects on electronics. In International Conference on Electromagnetics in Advanced Applications.

Deveci, B. M. (2007). Directed-Energy Weapons: Invisible and Invincible? Naval Postgraduate School, Monterey: Master of Science in Electronic Warfare Systems Engineering.

Feickert, A. (2016). Army and marine corps active protection systems (APS) efforts. In United States Congressional Research Service Report.

Feng, B., X. Liang, W. G. Du, C. L. Qiu, and R. S. Hou (2020). A stochastic vulnerability analysis method for armored vehicles with active protection systems. In Journal of Physics Conference Series, Volume 1507.

Graswald, M., R. Gutser, J. Breiner, F. Grabner, and T. Lehmann (2019). Defeating modern armor and protection systems. In Proceedings of the 2019 Hypervelocity Impact Symposium.

Hafften, M. and R. Stratton (2004). High energy laser weapon integration with ground vehicles. In NATO Report presented to RTO AVT Symposium, Volume RTO-MP-AVT-108.

Heiss, J., A. Kiser, E. M. Bouhafa, and S. Williams (2019). The combat cloud: Enabling multidomain command and control across the range of military operations. In Wright Flyer Paper No. 65, Air University Press.

Lavan, M. (2009). High energy laser systems for short range defence. In ACTA Physica Polonica A, Volume 115, pp. 959-963.

Meyer, T. J. (1998). Active protective systems: Impregnable armor or simply enhanced survivability? In Armor, Volume May-June.

Nielsen, P. E. (1994). Effects of Directed Energy Weapons. Washington: National Defence University.

Perry, W., R. W. Button, J. Bracken, T. Sullivan, and J. Mitchell (2002). Measures of Effectiveness for the Information-Age Navy: The Effects of Network-Centric Operations on Combat Outcomes. RAND, National Defense Research Institute.

Weinberg, G. V. (2021). Quantification of combat team survivability with high power RF directed energy weapons. Progress In Electromagnetics Research M 102, 1-11.

Weinberg, G. V. and M. M. Kracman (2021). Armoured fighting vehicle team performance prediction against missile attacks with directed energy weapons. Progress In Electromagnetics Research (under review).

Yang, L. and J. Xu (2020). Analysis on the development of active protection system for tanks and armored vehicles. In Journal of Physics Conference Series, Volume 1855. 